\section{Vol. 67, No. SS-1}

In the Surveillance Summary "Disparities in Preconception Health Indicators - Behavioral Risk Factor Surveillance System, 2013-2015, and Pregnancy Risk Assessment Monitoring System, 2013-2014," on page 7, the second sentence under the heading "Postpartum Use of Contraception (PRAMS)" should have read "The most effective methods (i.e., male or female sterilization, implant, and intrauterine device) have a failure rate that is $<1 \%$ with typical use, and moderately effective methods (shot, pill, patch, ring or diaphragm) include those with typical failure rates of $\mathbf{6 \% - 1 2 \% . " ~}$

\section{Vol. 67, No. 10}

In the report "Emergence of Monkeypox - West and Central Africa, 1970-2017," on page 306, the next to the last sentence in the first paragraph under "Monkeypox Cases in West Africa and Central Africa" should have read "With 89 confirmed cases, Nigeria is currently experiencing the largest documented outbreak of human monkeypox in West Africa." 